\title{
Inguinal hernia in a 55-year-old man
}

\author{
John M.A. Bohnen MD
} Competing interests: None
declared.

This article has been peer reviewed.

Correspondence to: John Bohnen, bohnenj@smh.ca

CMAJ 2014. DOI:10.1503 /cmaj.130909

\begin{abstract}
A 55-year-old man describes first feeling a painful lump on the left side of his groin while playing soccer. He says it does not interfere with his work as a carpenter, although he has stopped helping coworkers lift heavy objects. He smokes eight cigarettes per day. On examination, a tender inguinal hernia the size of a hen's egg is found on the left side above the inguinal ligament. The hernia reduces spontaneously when the patient lies down. He worries about how an operation might affect his sexual function.
\end{abstract}

\section{Should the patient undergo testing to confirm the diagnosis or characterize the hernia?}

No investigations are needed. Experts agree that when physical examination shows a diagnosis of inguinal hernia, ultrasonography or other diagnostic imaging is unnecessary. Guidelines advise against imaging a clinically evident hernia. ${ }^{1} \mathrm{~A}$ systematic review concluded that, although ultrasonography can confirm an obvious groin hernia, it is unnecessary in that setting and is an inefficient use of resources. ${ }^{2}$

If a primary care provider suspects a groin hernia but is uncertain, ultrasonography lacks sufficient accuracy to rule out a hernia with enough confidence to avoid referral to a surgeon. ${ }^{2,3}$ In such cases, a surgeon will usually diagnose or rule out a hernia on the basis of history and examination and will order imaging tests only if the patient has a convincing history

\section{Box 1: Choosing Wisely Canada recommendation*}

Avoid repair of minimally symptomatic inguinal hernias where appropriate by offering an option of watchful waiting for up to two years.

- Repair of minimally symptomatic inguinal hernias in adults can prevent potentially serious complications due to hernia incarceration. However, such repairs can also lead to complications such as infection, chronic inguinal pain and hernia recurrence, which cumulatively approximate the risks of incarceration. Evidence shows that such hernias can also be managed with watchful waiting for up to two years after assessment, ${ }^{5}$ a choice that should be offered to appropriately selected persons.

*Source: Canadian Association of General Surgeons: Six things physicians and patients

should question. Choosing Wisely Canada; 2014. Available: www.choosingwiselycanada.org /recommendations/canadian-association-of-general-surgeons-3/ and equivocal findings. The surgeon can determine at operation if the hernia is direct (medial inguinal floor defect) or indirect (more lateral defect, with peritoneal sac) and does not need to make that distinction in advance to plan treatment.

\section{Should the patient be referred to a surgeon?}

The patient should be referred to a surgeon to discuss treatment options. Repair is indicated for symptom relief and to prevent intestinal obstruction and strangulation. ${ }^{1}$ Where access to elective repair is limited, emergency surgery is more frequent and disabilities from untreated hernias have adverse economic consequences. ${ }^{4}$ Although two large, prospective, randomized trials showed that minimally symptomatic hernias could be managed initially without operation, ${ }^{5,6}$ about twothirds of the patients in the watchful-waiting arm in those studies eventually chose to have their hernias repaired, most commonly for pain relief. ${ }^{6,7}$ Even a patient with a minimally symptomatic inguinal hernia should be assessed by a surgeon to discuss treatment options, which may include watchful waiting for up to two years ${ }^{7}$ (Box 1).

\section{How urgent should the referral be?}

The degree of urgency depends on how much the hernia affects the patient and the extent to which a treatment delay increases the risk of complications. Our patient should be offered a timely, nonurgent referral because his symptoms affect his work capacity and his inguinal hernia reduces easily.

A review of data from multiple studies involving more than 200000 patients showed that the rate of death associated with emergency hernia repair for intestinal complications was about 20fold higher than the rate associated with elective surgery (4\% v. $0.2 \%) .{ }^{8}$ Although unnecessary delay should be avoided, the risk of a bad outcome while awaiting hernia repair is low among patients with minimal symptoms, who require emergency surgery at a frequency of less than $0.5 \%$ annually. ${ }^{7,8}$ Less is known about symptom- 
atic hernias, which are usually repaired, which precludes their inclusion in long-term follow-up studies. A useful retrospective analysis pointed out challenges estimating such risks and identified femoral and scrotal hernias among factors associated with the need for emergency repair. ${ }^{9}$

Femoral hernia, which may present acutely with intestinal obstruction, or less dramatically as a lump or swelling inferior to the inguinal ligament, accounts for a small (1\%) subset of groin hernias among men and $22 \%$ of groin hernias among women, according to a Swedish hernia registry study involving more than 100000 patients. ${ }^{10}$ Femoral hernias are associated with higher rates of hernia complications, intestinal resection and death after emergency repair compared with other groin hernias, but not higher mortality when repaired electively. ${ }^{10,11}$

\section{What should the patient be told while waiting for the referral appointment?}

The patient should be instructed to seek emergency medical help if symptoms and signs of intestinal obstruction or strangulation develop. He can expect that surgery will fix his hernia, with less than $5 \%$ risk of hernia recurrence, infection or severe chronic pain, although wide variations in these adverse outcomes have been reported in the literature. ${ }^{8}$ He can improve his chances for success by stopping smoking. Smoking increases the risk of postoperative infection and hernia recurrence. ${ }^{12}$

Open and laparoscopic repairs produce similarly good results and usually do not require an overnight stay in hospital. Open repairs can be performed often under local anesthesia. Depending on the circumstances, return to work in two weeks and resumption of heavy exertion in three weeks are reasonable expectations. ${ }^{1}$ Surgical repair should not impair the patient's sexual function, which improves often after repair of large hernias. ${ }^{13}$

\section{Case revisited}

The patient stopped smoking, assisted by medication prescribed by his primary care provider. After elective hernia repair under local anesthesia and sedation, he resumed work two weeks later and full activity, including soccer and heavy lifting, three weeks after surgery.

\section{References}

1. Simons MP, Aufenacker T, Bay-Nielsen M, et al. European Hernia Society guidelines on the treatment of inguinal hernia in adult patients. Hernia 2009;13:343-403.

2. Robinson A, Light D, Nice C. Meta-analysis of sonography in the diagnosis of inguinal hernias. J Ultrasound Med 2013;32:339-46.

3. Light D, Ratnasingham K, Banerjee A, et al. The role of ultrasound scan in the diagnosis of occult inguinal hernias. Int J Surg 2011;9:169-72.

4. Ohene-Yeboah M, Abantanga F, Oppong J, et al. Some aspects of the epidemiology of external hernias in Kumasi, Ghana. Hernia 2009; 13:529-32.

5. Fitzgibbons RJ Jr, Giobbie-Hurder A, Gibbs JO, et al. Watchful waiting vs repair of inguinal hernia in minimally symptomatic men: a randomized clinical trial. JAMA 2006;295:285-92.

6. Chung L, Norrie J, O'Dwyer P. Long-term follow-up of patients with a painless inguinal hernia from a randomized clinical trial. Br J Surg 2011;98:596-9.

7. Fitzgibbons RJ Jr, Ramanan B, Arya S, et al. Long-term results of a randomized controlled trial of a nonoperative strategy (watchful waiting) for men with minimally symptomatic inguinal hernias. Ann Surg 2013;258:508-15.

8. INCA Trialists Collaboration. Operation compared with watchful waiting in elderly male inguinal hernia patients: a review and data analysis. J Am Coll Surg 2011;212:251-9.e1-4

9. Abi-Haidar Y, Sanchez V, Itani KMF. Risk factors and outcomes of acute versus elective groin hernia surgery. J Am Coll Surg 2011;213:363-9.

10. Nilsson H, Stylianidis G, Haapamaki M, et al. Mortality after groin hernia surgery. Ann Surg 2007;245:656-60.

11. Rai S, Chandra SS, Smile SR. A study of the risk of strangulation and obstruction in groin hernias. Aust N Z J Surg 1998;68:650-4.

12. Sørensen LT. Wound healing and infection in surgery. The clinical impact of smoking and smoking cessation: a systematic review and meta-analysis. Arch Surg 2012;147:373-83.

13. Ertan T, Keskek M, Kilic M, et al. Recovery of sexual function after scrotal hernia repair. Am J Surg 2007;194:299-303.

Affiliations: Department of Surgery, St. Michael's Hospital; Faculty of Medicine, University of Toronto, Toronto, Ont.

CMAJ is collaborating with Choosing Wisely Canada, with support from Health Canada, to publish a series of articles describing how to apply the Choosing Wisely Canada recommendations in clinical practice. 